\title{
INSUFFICIENCIES OF THE SINGLE EXPONENTIAL MODEL AND EFFICIENCY OF THE DOUBLE EXPONENTIAL MODEL IN THE OPTIMIZATION OF SOLAR CELLS EFFICIENCY
}

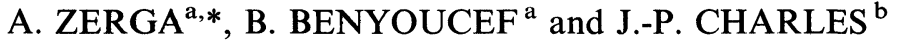 \\ ${ }^{a}$ Université de Tlemcen, Institut de Physique, Laboratoire de Physique \\ Energétique et Matériaux, B.P.: 119, 13000 Tlemcen, Algérie; \\ ${ }^{\mathrm{b}}$ Centre Lorrain d'Optique et d'Electronique des Solides, Supelec, \\ 2 rue Edouard Belin, 57078 Metz Cedex 3, France
}

(Received 7 July 1998; In final form 4 September 1998)

\begin{abstract}
Single and double exponential models are confronted to determine the most adapted model for optimization of solar cells efficiency. It is shown that the single exponential model (SEM) presents some insufficiencies for efficiency optimization. The interest of the double exponential model to optimize the efficiency and to achieve an adequate simulation of the operation of solar cells is demonstrated by means of $I-V$ characteristics plotting.
\end{abstract}

Keywords: Single exponential model; double exponential model; optimization; solar cells efficiency; restrictive parameters

\section{INTRODUCTION}

Many authors tried to put in evidence the restrictive parameters of the solar cell efficiency [1-3]. The detailed knowledge of these limiting parameters and the evolution of the technology permitted to increase the silicon solar cell efficiency from $6 \%$ in 1954 at the Bell Laboratory to a value greater than $24 \%$ in 1990 at the University of New South Wales [4].

\footnotetext{
*Corresponding author.
} 
In most of these qualitative studies, the single exponential model was used to describe the $I-V$ solar cells characteristics. Using this model permits to show, for a solar cell family characterized by the restricted values of the open circuit voltage, that the ideal efficiency is maximal when the diode ideality factor is equal to unity and the saturation current is minimal. This does reflect the capacity of the model to describe the $I-V$ characteristics only if the influence of the diffusion current in the quasi neutral regions is preponderant over the recombination current in the space charge region. Whenever this predominance is not satisfied, the double exponential model must be used for an adequate description of the $I-V$ characteristics.

With the single exponential model, for large values of the diode ideality factor, the possibility of over estimation of the efficiency occurs for open-circuit voltage values superior to the intrinsic limit of the silicon solar cell which are incompatible with the present technology.

This study provides a justification in describing the operation of solar cells by representing separately the contributions of the diffusion current in the quasi-neutral regions and the recombination current in the space-charge region, for searching an optimal efficiency value.

\section{METHOD FOR MAXIMUM EFFICIENCY DETERMINATION}

For a solar cell $I-V$ characteristics described by the single or double exponential models, the output power $P$ is a function of current $I$ and voltage $V$ :

$$
P(I, V)=I \cdot V
$$

The maximum values of current and voltage, $I_{M}$ and $V_{M}$ respectively, corresponding to the maximum power $\mathrm{PM}\left(\mathrm{PM}=I_{M} \cdot V_{M}\right)$, cannot be obtained by resolution of the implicit equation of the $I-V$ characteristics only:

$$
\left\{\begin{array}{c}
I=h(I, V) \\
I=I_{p h}-\frac{V+R_{s} I}{R_{s h}}-\sum_{i=1,2} I_{o i}\left[\exp \left(\beta_{i}\left(V+R_{s} I\right)-1\right]\right. \\
\quad \text { where } \beta_{1}=\frac{q}{n k T}, \quad \beta_{2}=\frac{q}{k T}
\end{array}\right.
$$


where $i=1$ in the single exponential model, $i=2$ in the double exponential model, and $I_{p h}, R_{s}, R_{s h}, I_{o i}$ and $n_{i}$ are the parameters of the model which are related to the internal properties of the solar cell.

Consequently, solving a second equation is fundamental. This second equation can be obtained by applying the method of Lagrange [5] to an object function of two variables $P(I, V)$ bounded by the implicit equation of the $I-V$ characteristics. Let a problem $(\mathcal{P})$, be:

$$
(\mathcal{P}) \quad\left\{\begin{array}{l}
\min (-P) \\
H(I, V)=0 \quad \text { where } \quad H(I, V)=I-h(I, V)
\end{array}\right.
$$

where $H$ represents equality constraints. We associate to this problem $(\mathcal{P})$ the Lagrangian $L$ defined as:

$$
L(I, V, \mu)=-P(I, V)+\mu^{T} \cdot H(I, V)
$$

where $\mu$ are Lagrange multipliers associated to the constraints of equality. The necessary conditions of optimality so that $X^{*}$ be a minimum, are written:

$$
\left\{\begin{array}{l}
\text { 1) } \nabla L=0 \\
\text { 2) } \mu^{T} \cdot H(I, V)=0 \quad(\mu \in R)
\end{array}\right.
$$

After calculating the partial derivatives of the $L$ function, we obtain the following implicit equation:

$$
I=\left(V-R_{s} I\right)\left[\frac{1}{R_{s h}}+\sum_{i} I_{o i} \beta_{i} \exp \left(\beta_{i}\left(V+R_{s} I\right)\right)\right]
$$

So we get a system of two non linear equations with two variables $I$ and $V$

$$
\left\{\begin{array}{l}
H(I, V)=I-I_{p h}+\frac{V+R_{s} I}{R_{s h}}+\sum_{i} I_{o i}\left(\exp \left(\beta_{i}\left(V+R_{s} I\right)\right)-1\right) \\
G(I, V)=I-\left(V-R_{s} I\right)\left[\frac{1}{R_{s h}}+\sum_{i} I_{o i} \beta_{i}\left(\exp \left(\beta_{i}\left(V+R_{s} I\right)\right)\right)\right]
\end{array}\right.
$$

For a set of parameters of the given model, the numeric resolution of this system allows us to determine the value of the maximum power delivered and consequently the maximum efficiency $\eta_{M}\left(\eta_{M}=P_{M} / P_{0}\right)$, where $P_{0}$ represent the incidental power. 


\section{OPTIMIZATION OF THE EFFICIENCY OF A SOLAR CELL - RESULTS AND DISCUSSION}

For given operating conditions, the study of the influence of the parameters of a single and double exponential models $\left(I_{p h}, R_{s}\right.$, $R_{s h}, I_{01}, I_{02}$ and $n_{1}$ ) on the value of the optimal efficiency of a solar cell requires a complete analytic model.

In this study, we chose very general variational ranges for these parameters (Tab. I) by assuming an incident luminous power of $100 \mathrm{mw} \cdot \mathrm{cm}^{-2}$ distributed over the whole spectrum and absorbed completely by the solar cell.

\subsection{Optimizing with the Single Exponential Model}

For a set of given parameters, we look for the maximal value of the efficiency $\eta_{M}\left(R_{s}, R_{s h}, n, I_{p h}\right.$ and $\left.I_{01}\right)$ by varying only one parameter, the others being fixed to their extremal values. Table II shows results of these variations: The weak influence of $R_{s}, R_{s h}, I_{p h}$, and the large influence of $n, I_{p h}$ and $I_{01}$ on the values of $\eta_{M}$ and $V_{\mathrm{oc}}$ are to be noted. This allows us to fix $R_{s}, R_{s h}$ and $I_{p h}$ to their extremal values $\left(R_{s}=0.1 \Omega ; R_{s h}=10^{4} \Omega\right.$ and $\left.I_{p h}=0.04 \mathrm{~A} \cdot \mathrm{cm}^{-2}\right)$ which are compatible with present technology [6].

The results in Table II show also the strong variations in the opencircuit voltage $V_{\text {oc }}$ with the diode ideality factor $n$ and the saturation current $I_{01}$; this open-circuit voltage is inherent to the design of solar cells and its determination allows us to select the optimal parameters calculated according to the possibility of technological realization. $V_{\mathrm{oc}}$ is evaluated from the implicit equation $I=h(I, V)$ for a zero current $(I=0)$ and is bound to the parameters of the model by the implicit

TABLE I Variation ranges of parameters of single and double exponential model of $I-V$ characteristics

\begin{tabular}{lc}
\hline Single exponential model & Double exponential model \\
\hline$n \in[1-2.4]$ & $n \in[1.3-2.7]$ \\
$R_{s} \in[0.1-0.4] \Omega$ & $R_{s} \in[0.1-0.4] \Omega$ \\
$R_{s h} \in\left[400-10^{4}\right] \Omega$ & $R_{s h} \in\left[400-10^{4}\right] \Omega$ \\
$I_{p h} \in[0.02-0.04] \mathrm{A} \cdot \mathrm{cm}^{-2}$ & $I_{p h} \in[0.02-0.038] \mathrm{A} \cdot \mathrm{cm}^{-2}$ \\
$I_{01} \in\left[10^{-9}-10^{-7}\right] \mathrm{A} \cdot \mathrm{cm}^{-2}$ & $I_{01} \in\left[10^{-9}-10^{-5}\right] \mathrm{A} \cdot \mathrm{cm}^{-2}$ \\
& $I_{02} \in\left[10^{-13}-10^{-9}\right] \mathrm{A} \cdot \mathrm{cm}^{-2}$ \\
\hline
\end{tabular}




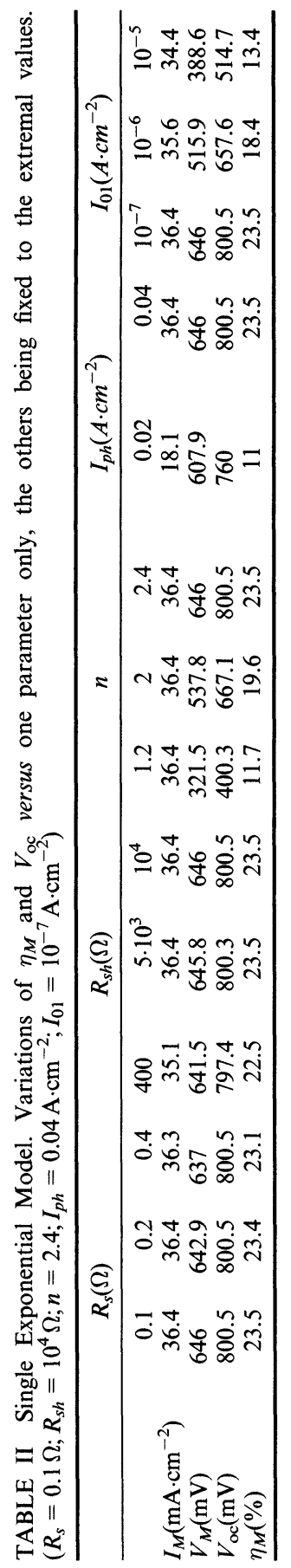


equation:

$$
V_{\mathrm{oc}}=R_{s h}\left[I_{p h}+\sum_{i} I_{o i}\left(1-\exp \beta_{i} V_{\mathrm{oc}}\right)\right]
$$

Figure 1 show variations of the maximal efficiency $\eta_{M}$ and of the opencircuit voltage $V_{\text {oc }}$ against (a) the saturation current $I_{01}$ and (b) the diode ideality factor $n$.

In these figures, we notice that optimal efficiency $\eta_{M}$ and open-circuit voltage $V_{\text {oc }}$ variations are in interrelationship. Conditions on $n$ and $I_{01}$ permitting to reach large values of $\eta_{M}$ impose large values of $V_{o c}$.

This model foresees an increase of $V_{\mathrm{oc}}$ as soon as the diode ideality factor increases or the saturation current decreases, it leads to large values of $V_{\text {oc }}$, not practicable. Consequently, a research for new structures must not be carried solely in view of the improvement of the saturation current $I_{01}$ of the ideal solar cell but rather in view of an improvement of $V_{\text {oc }}$ with the diode ideality factors greater than unity. Also, these results prove the existence of a non negligible recombination current in the space-charge region for large values of $V_{\text {oc }}$ related to very deep traps and to high $n$ values.

This shows the insufficiency of the single exponential model and justifies the interest of using the double exponential model in order to be able to take into account the two phenomena, i.e., the diffusion and the recombination in space-charge region, in the optimization of efficiency. This optimization is performed for a set of parameters of the model.

\subsection{Optimization with the Double Exponential Model}

In this model, the optimal value of the maximal efficiency $\eta_{M}$ is obtained again for the extreme values of parameters of this model, minimal for $R_{s}, I_{01}$ and $I_{02}$ and maximal for $I_{p h}, R_{s h}$ and $n$. We fix the parameters, $R_{s}, R_{s h}$ and $I_{p h}$ to their values of the maximal efficiency $\left(R_{s}=0.1 \Omega ; R_{s h}=10^{3} \Omega\right.$ and $\left.I_{p h}=0.038 \mathrm{~A} \cdot \mathrm{cm}^{-2}\right)$ and we study the influence of variations of $n, I_{01}$ and $I_{02}$ on the maximal efficiency $\eta_{M}$ and on the open-circuit voltage $V_{\text {oc }}$ (Tab. III). We notice that for a given value of the diode ideality factor $n$, the influence of the diffusion current $I_{02}$ is predominant over the recombination current in space- 

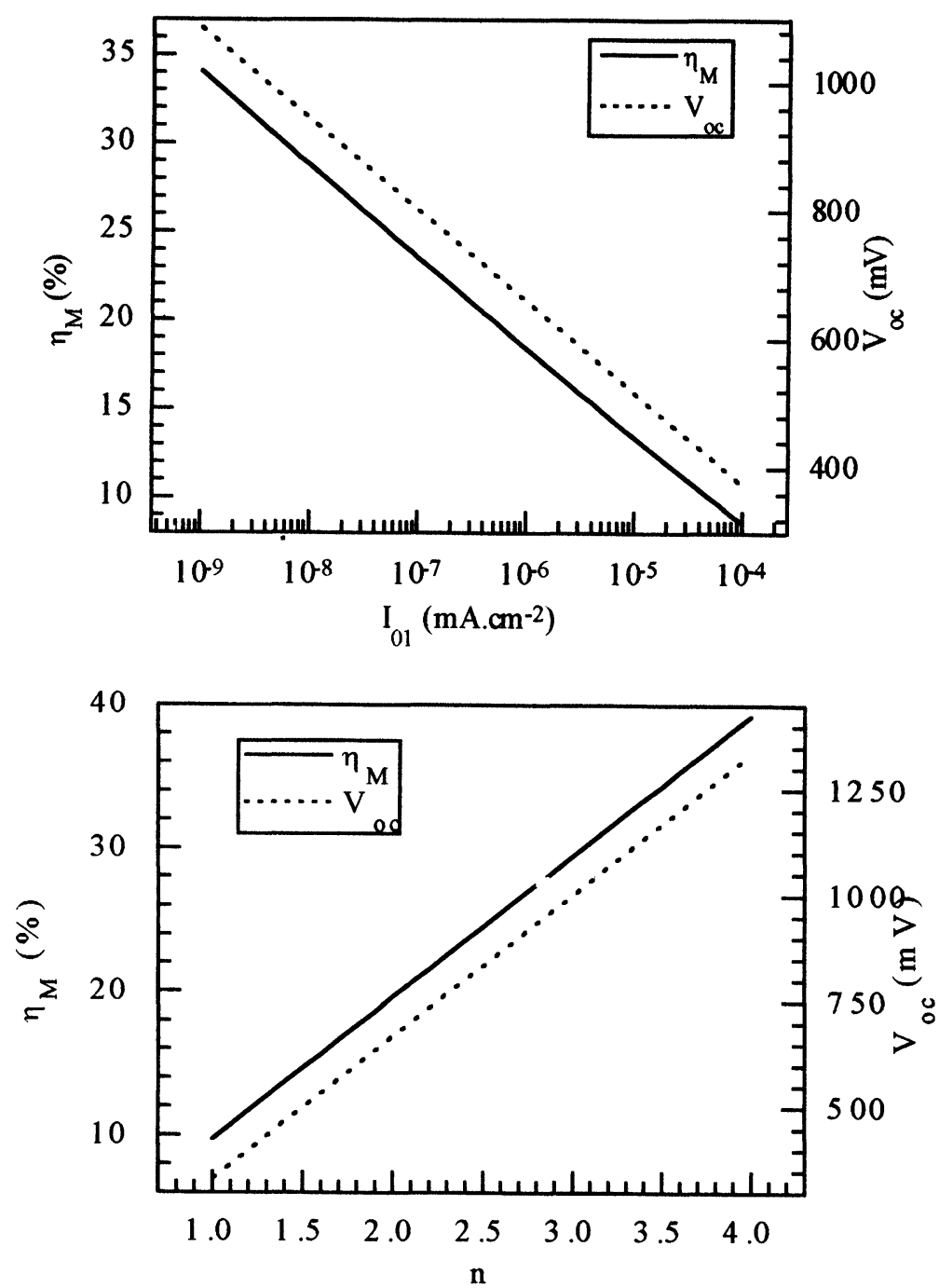

FIGURE 1 Single Exponential Model. Variations of $\eta_{M}$ and $V_{\text {oc }}$ versus $I_{01}$ and $n$. $\left(R_{s}=0.1 \Omega ; R_{s h}=10^{4} \Omega ; n=2.4 ; I_{p h}=0.04 \mathrm{~A} \cdot \mathrm{cm}^{-2} ; I_{01}=10^{-7} \mathrm{~A} \cdot \mathrm{cm}^{-2}\right)$.

charge region $I_{01}$. For a large value of the diode ideality factor, large values of efficiency $\eta_{M}$ can be obtained with an open-circuit voltage value compatible with the present technological realizations for $V_{\text {oc }}(\cong 0.690 \mathrm{~V})[7]$. 


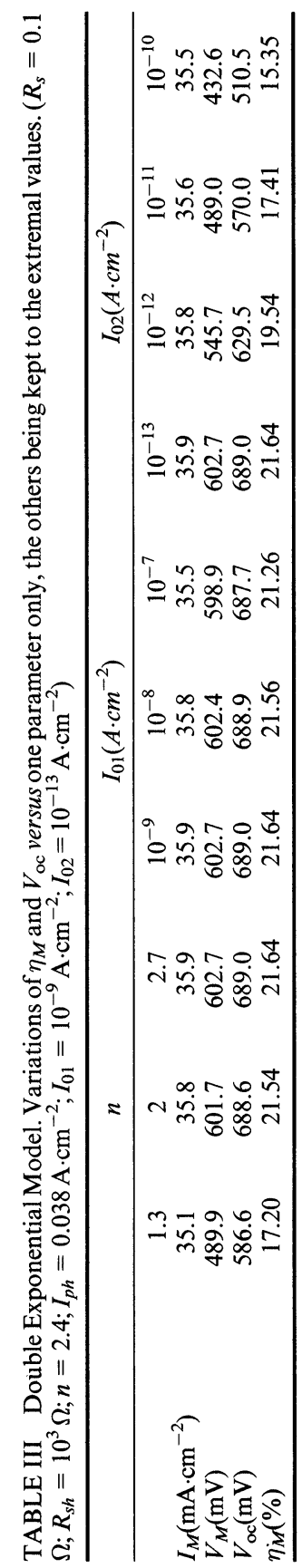


Figure 2 present variations of the maximal efficiency $\eta_{M}$ and of the open-circuit voltage $V_{\text {oc }}$ against (a) the saturation currents $I_{01}$ and $I_{02}$, and (b) the diode ideality factor $n$. We notice that for a recombination current $I_{01}$ of the order of $10^{-9} \mathrm{~A} \cdot \mathrm{cm}^{-2}$ and a diffusion current $I_{02}$ of
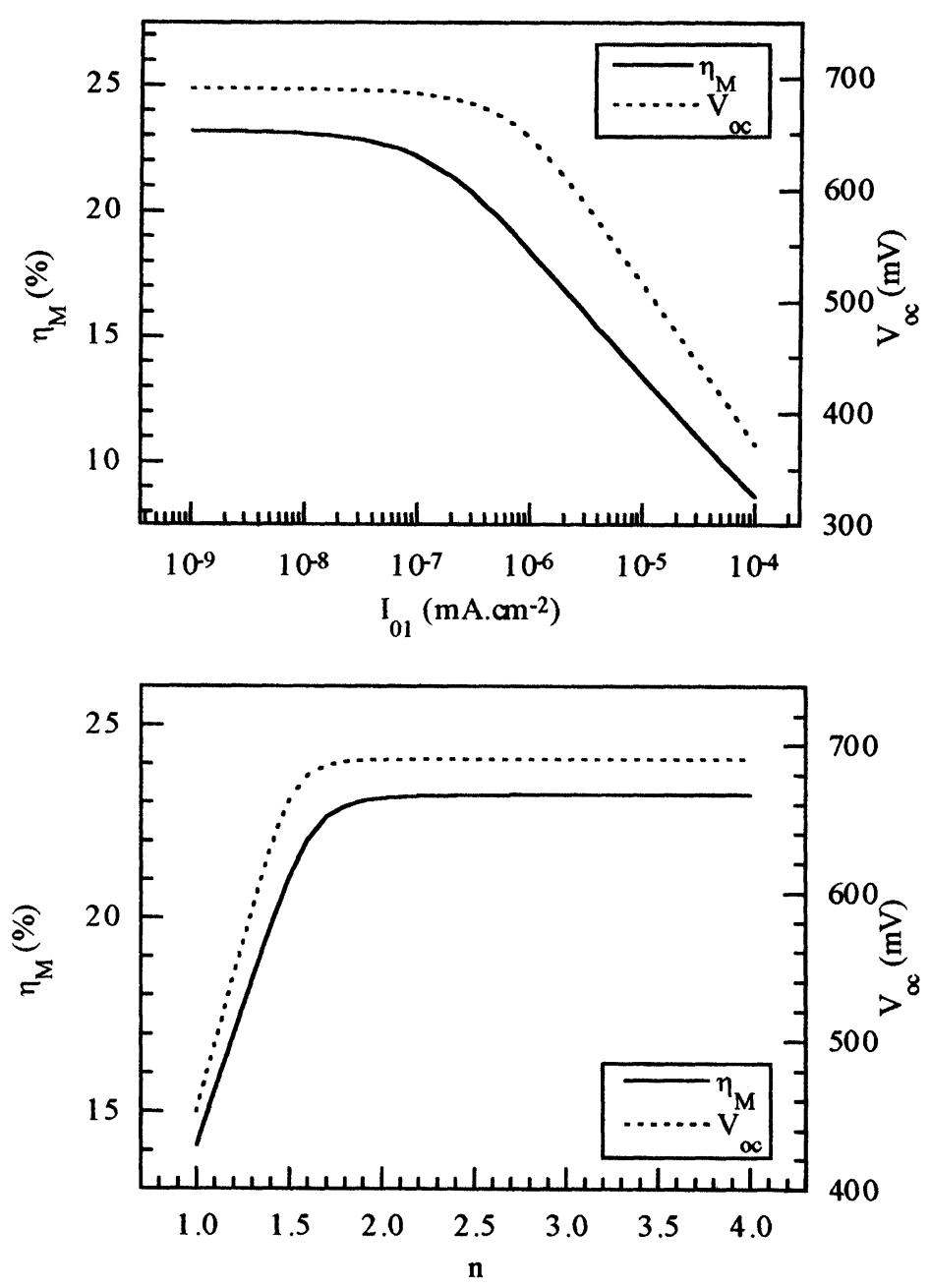

FIGURE 2 Double exponential model. Variations of $\eta_{M}$ and $V_{\text {oc }}$ versus $I_{01}$ and $n$. $\left(R_{s}=0.1 \Omega ; R_{s h}=10^{3} \Omega ; n=2.7 ; I_{p h}=0.038 \mathrm{~A} \cdot \mathrm{cm}^{-2} ; I_{01}=10^{-9} \mathrm{~A} \cdot \mathrm{cm}^{-2} ; I_{02}=10^{-13}\right.$ A $\left.\cdot \mathrm{cm}^{-2}\right)$. 
$10^{-13} \mathrm{~A} \cdot \mathrm{cm}^{-2}$, the optimal efficiency and open-circuit voltage reach their maximal values for a diode ideality factor greater than 2 . This result shows the importance of the type and the position of trap levels in the determination of $\eta_{M}$ and $V_{\text {oc }}$ since the maximum of the recombination current in the space charge region is found for trap levels situated in the middle of the forbidden band $(n=2)$.

Figure 3 presents variations of the maximal efficiency against recombination $I_{01}$ and diffusion $I_{02}$ currents, for different values of $n$. For low values of the diode ideality factor $n$, the influence of the recombination current $I_{01}$ is very strong. Above $n=1.7$, the phenomenon is reversed and the diffusion current $I_{02}$ has a strong influence, while that of the recombination current $I_{01}$ lowers. Finally, a good value of the efficiency can be obtained for a high diode ideality factor and an important recombination current in the space-charge region with the condition that the diffusion current is minimized. This result is very important in the design of solar cells.

\subsection{Comparison Between Single and Double Exponential Models of the $I-V$ Characteristics}

To illustrate both the insufficiency of the single exponential model and the interest to use the double exponential model in the optimization and in the simulation of the operation of a solar cell characterized by a set of optimal parameters, we present the simulated $I-V$ characteristics.

Figures 4 and 5 show variations of the $I-V$ characteristics according to the diode ideality factor $n$ for the single and double exponential models, respectively. For the two models, we note that the $I-V$ characteristics are determined mainly by dark currents $I_{\mathrm{d} 1}\left(I_{\mathrm{d} 1}=\right.$ $\left.I_{01}\left\lfloor e^{\beta_{1}\left(V+R_{s} I\right)}-1\right\rfloor\right)$ and $I_{\mathrm{d} 2}\left(I_{\mathrm{d} 2}=I_{02}\left\lfloor e^{\beta_{2}\left(V+R_{s} I\right)}-1\right\rfloor\right)$.

On the other hand, the $I_{p h}$ photocurrent and the shunt current $I_{R_{s h}}\left(I_{R_{s h}}=\left(V+R_{s} I\right) / R_{s h}\right)$ present only weak variations.

For the single exponential model, the more the diode ideality factor increases, the more the $I-V$ characteristics expands. This produces important efficiencies, but with open-circuit voltage values incompatible not only with the present technologies but also with the intrinsic limit of $V_{\text {oc }}$ corresponding to silicon solar cells.

In the double exponential model, for $n$ greater than 1.7, we observe a superposition of the $I-V$ characteristics and therefore a saturation 

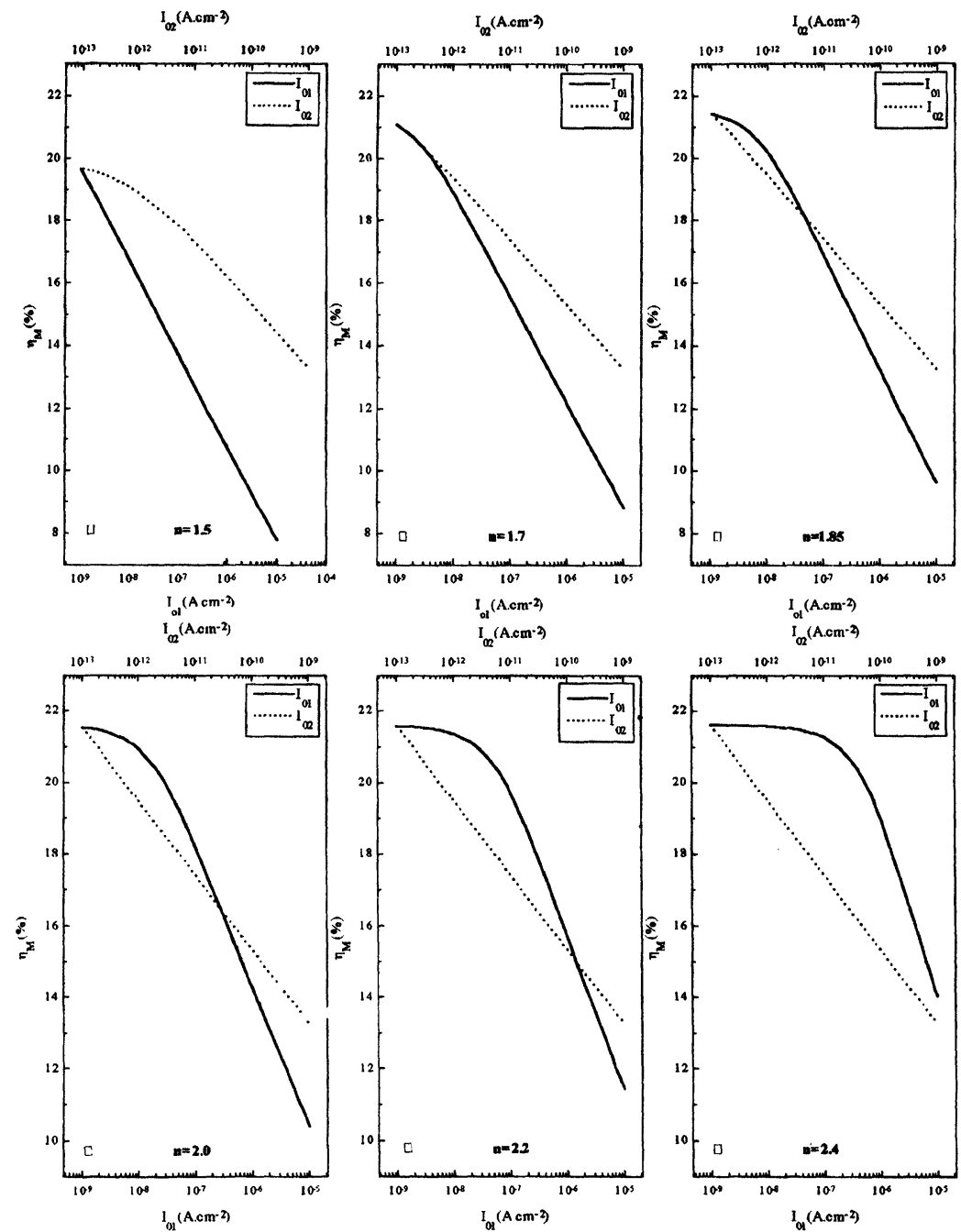

FIGURE 3 Variation of $\eta_{M}$ and $V_{\text {oc }}$ versus $I_{01}$ (with $I_{02}=10^{-13} \mathrm{~A} \cdot \mathrm{cm}^{-2}$ ) and $I_{02}$ (with $\left.I_{01}=10^{-9} \mathrm{~A} \cdot \mathrm{cm}^{-2}\right)$ for different values of $n .\left(R_{s}=0.1 \Omega ; R_{s h}=10^{3} \Omega ; I_{p h}=0.038\right.$ $\mathrm{A} \cdot \mathrm{cm}^{-2}$ ).

of the efficiency and of the open-circuit voltage to a value of $0.690 \mathrm{~V}$ acceptable physically and compatible with present technology. Another important result can be noted for low values of $n(n<1.8)$. Therefore, the influence of the dark current $I_{d 1}$ on the $I-V$ 


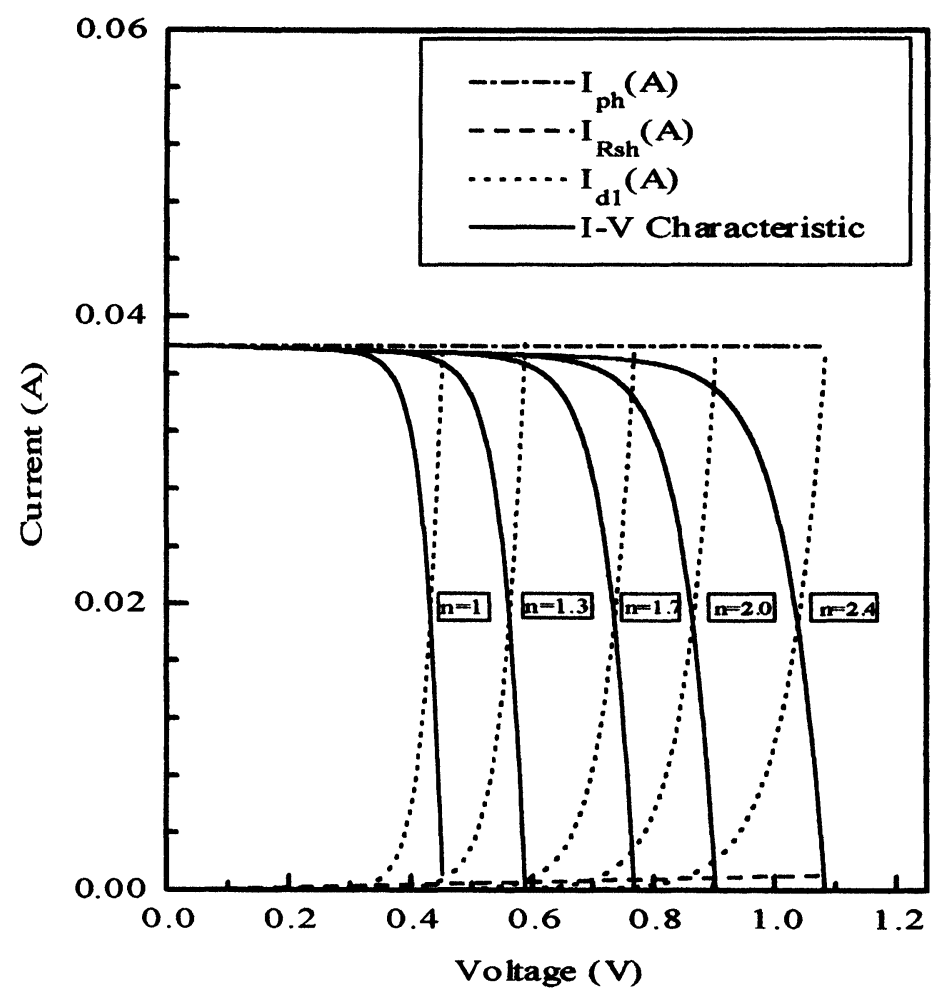

FIGURE $4 I-V$ Characteristics according to the single exponential model for different values of the diode ideality factor $n . \quad\left(R_{s}=0.1 \Omega ; R_{s h}=10^{4} \Omega ; I_{p h}=0,04 \mathrm{~A} \cdot \mathrm{cm}^{-2}\right.$; $I_{01}=10^{-9} \mathrm{~A} \cdot \mathrm{cm}^{-2}$.

characteristics would be more preponderant than that of the dark current $I_{\mathrm{d} 2}$.

For large values of $n(n>1.8)$, the phenomenon is reversed and it is the influence of $I_{\mathrm{d} 2}$ that becomes more preponderant than that of $I_{\mathrm{d} 1}$. This is explained physically by the fact that phenomena of recombination in space-charge region limit the performances of solar cell with low diode ideality factors, whereas the phenomena of recombination in quasi-neutral regions limit the performance of solar cells with large diode ideality factors. Finally, these two phenomena are of comparable importance and a determination of the optimal efficiency must take into account these two phenomena. 


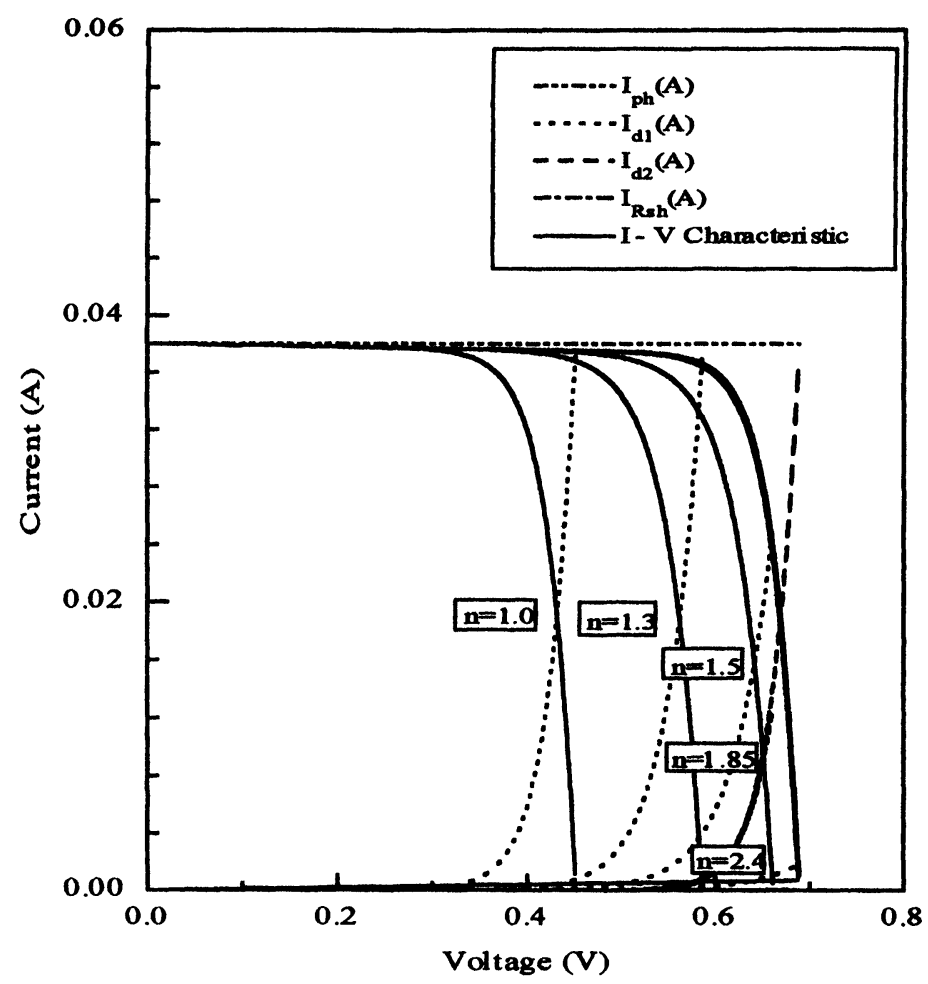

FIGURE $5 I-V$ Characteristics according to the double exponential model for different values of the diode ideality factor $n$. $\left(R_{s}=0.1 \Omega ; R_{s h}=10^{3} \Omega ; I_{p h}=0,038\right.$ $\mathrm{A} \cdot \mathrm{cm}^{-2} ; I_{01}=10^{-9} \mathrm{~A} \cdot \mathrm{cm}^{-2} ; I_{02}=10^{-13} \mathrm{~A} \cdot \mathrm{cm}^{-2}$.

\section{CONCLUSION}

This study puts into evidence the insufficiency of the single exponential model for large values of the diode ideality factor. This model leads to solar cells characterized by an open-circuit voltage larger than the physical limit and incompatible with present technology.

This study shows also the interest of using the double exponential model for optimization of the efficiency and for the simulation of solar cell operation. It also shows that this model permits to take into account recombination phenomena in the space-charge region and recombination in the quasi-neutral regions, and to make an adequate 
simulation of the operation of the solar cell. So, through this model, we can physically and technologically design solar cells with high efficiencies and acceptable values of the open-circuit voltage.

\section{References}

[1] Sah, C. T. (1986). Solar Cells, 17, 1-27.

[2] Redfield, D. (1980). IEEE Trans. on Elec. Devices, ED-27(4), 766-771.

[3] Hauser, J. R. and Dunbar, P. M. (1977). IEEE Trans. on Elec. Devices, ED-24(4), $305-321$.

[4] Wang, A., Zhao, J. and Green, M. A. (1990). Appl. Phys. Lett., 57(6), 602-604.

[5] Gill, E. P., Walter, M. and Wright, M. M. (1981). Practical Optimization, Academic Press, INC (London), LTD.

[6] Pelanchon, F., Mialhe, P. and Charles, J.-P. (1988). Revue de Phys. Appl., 23, 11391145.

[7] Green, M. A., Blakers, A. W., Shi, J., Killer, E. M. and Wenham, S. R. (1984). IEEE Trans. on Elec. Devices, ED-31(5), 679-683. 

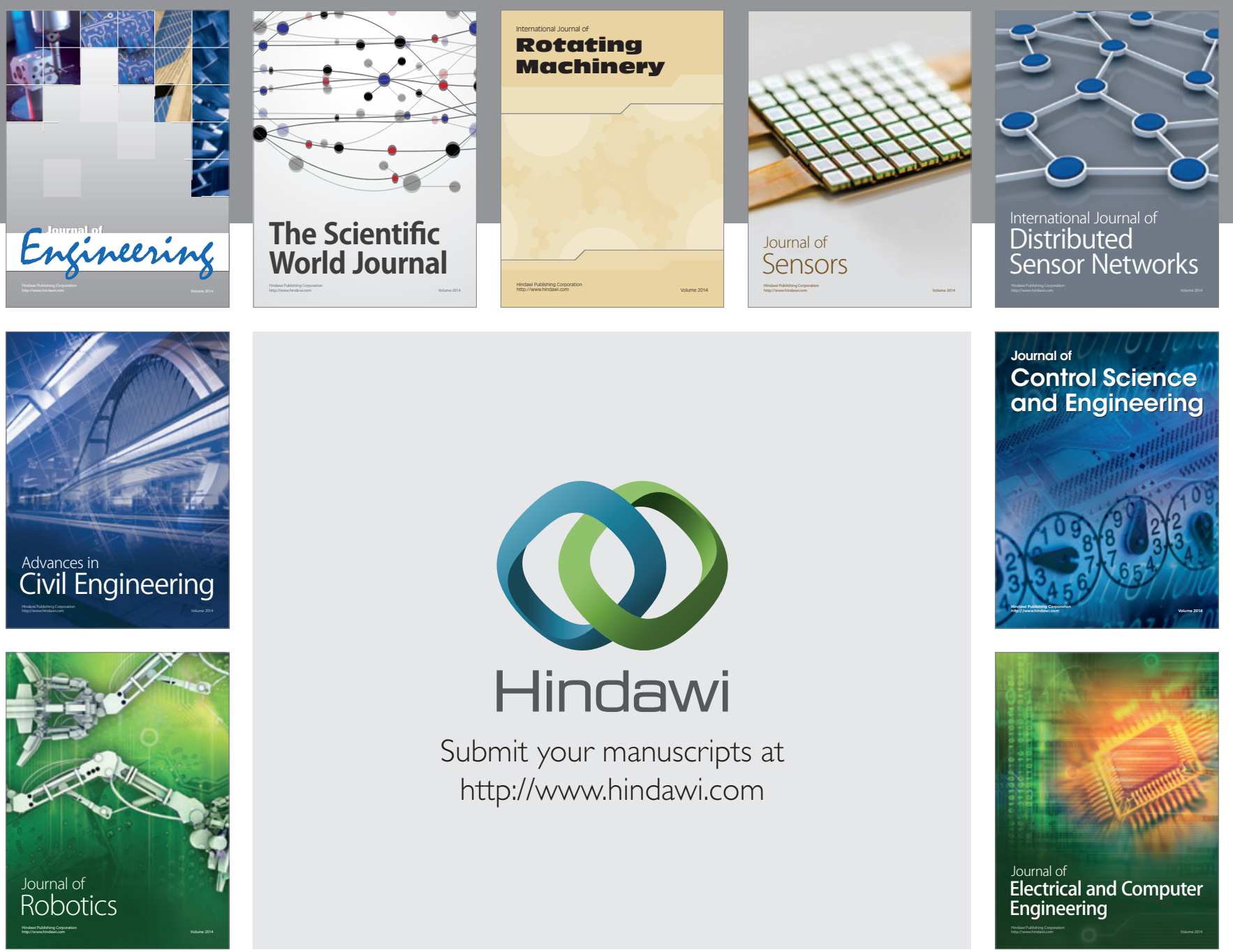

Submit your manuscripts at

http://www.hindawi.com
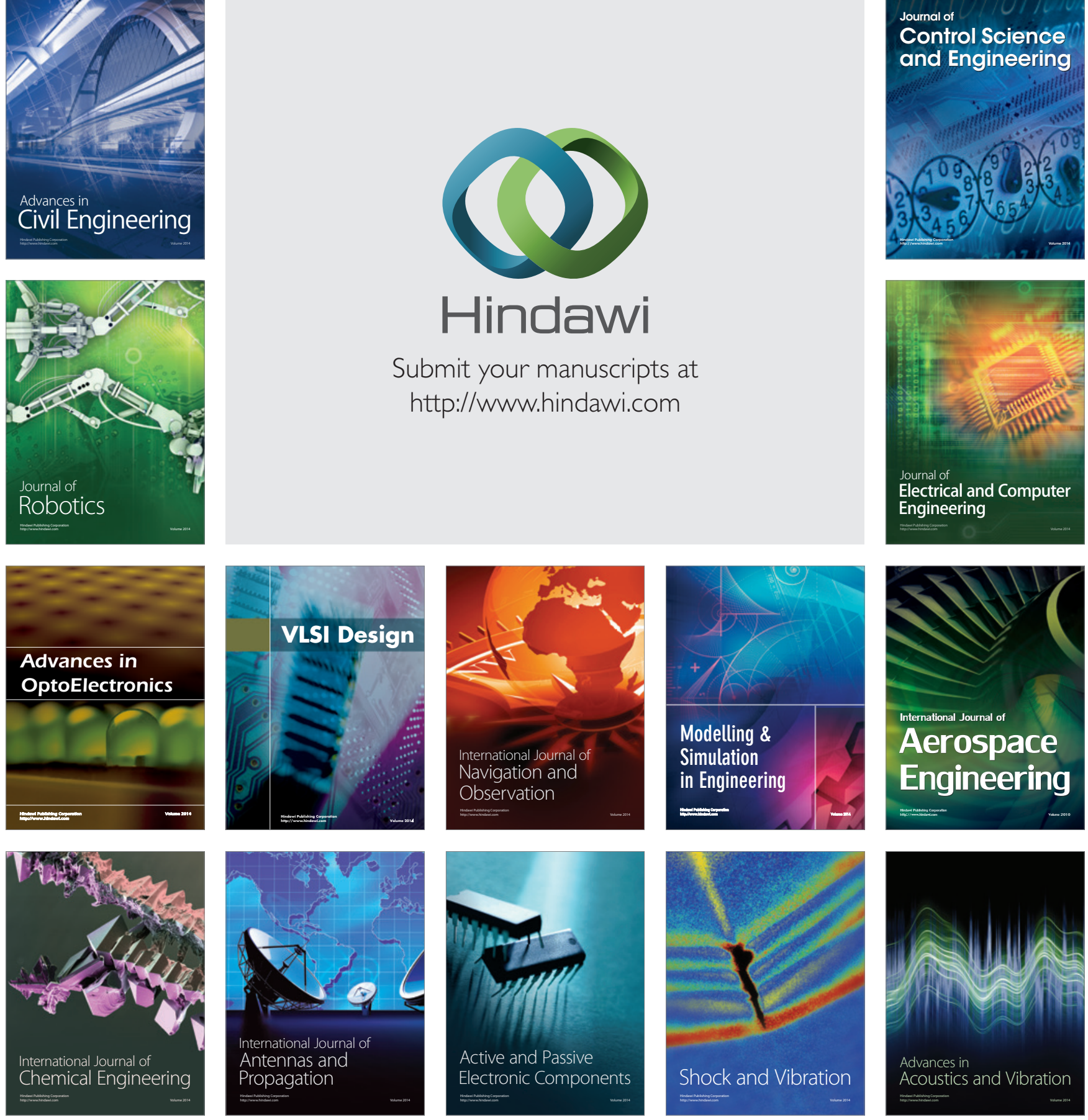